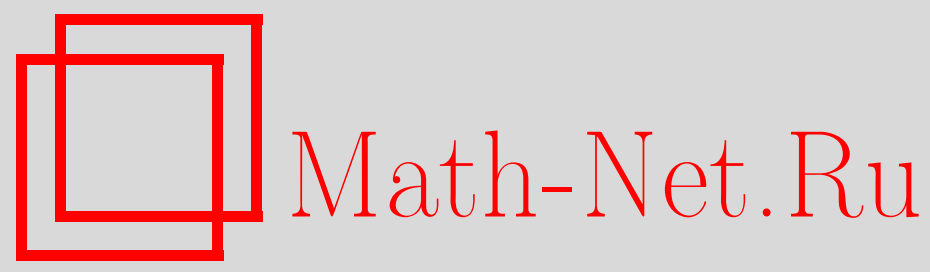

Е. Г. Зеленюк, Топологические группы, в которых каждое нигде не плотное подмножество замкнуто, Матем. заметки, 1998, том 64, выпуск 2, 207-211

DOI: https://doi.org/10.4213/mzm1387

Использование Общероссийского математического портала Math-Net.Ru подразумевает, что вы прочитали и согласны с пользовательским соглашением http://www.mathnet.ru/rus/agreement

Параметры загрузки:

IP : 54.172 .240 .79

26 апреля 2023 г., 05:40:15

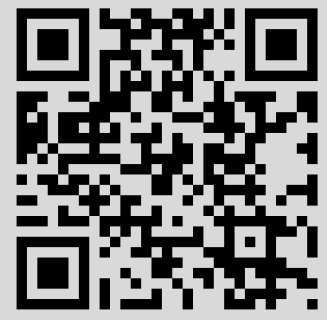




\title{
ТОПОЛОГИЧЕСКИЕ ГРУППЫ, В КОТОРЫХ КАЖДОЕ НИГДЕ НЕ ПЛОТНОЕ ПОДМНОЖЕСТВО ЗАМКНУТО
}

\author{
Е.Г. Зеленюк
}

\begin{abstract}
В предположении комбинаторного принципа $p=\mathfrak{C}$, следующего из аксиомы Мартина, доказано, что произвольная недискретная метризуемая групповая топология на абелевой группе усиливается до недискретной групповой топологии, в которой каждое нигде не плотное подмножество замкнуто.
\end{abstract}

Библиография: 9 названий.

Из экстремальных свойств топологических пространств достаточно известны экстремальная несвязность, неразложимость и замкнутость каждого дискретного подмножества. Пространство экстремально несвязно, если замькание любого открытого подмножества открыто. Пространство неразложимо, если его нельзя разбить на два плотных подмножества. Для каждого из перечисленных свойств существуют недискретные топологические групш, им обладаюшие, однако при дополнительных теоретико-множественных предположениях [1]-[3]. Недавно И. В. Протасов доказал, что "наивно" построить недискретную неразложимую топологическую абелеву группу невозможно. Проблема построения "наивньх" примеров счетной недискретной экстремально несвязной топологической группы и счетной недискретной топологической группы, в которой каждое дискретное подмножество замкнуто, остается открытой [4, I.16], [5].

Экстремальная несвязность и неразложимость топологической группы - достаточно жесткие ограничения на ее алгебраическое строение. Недискретная экстремально несвязная топологическая группа содержит открытую булеву подгруппу [2]. Булевой назьвается групша периода 2. Недискретная неразложимая топологическая абелева группа содержит открытую счетную булеву подгруппу [6]. Имеет ли место аналогичный результат для топологических групп, в которых каждое дискретное подмножество замкнуто, было неизвестно.

В данной заметке в предположении комбинаторного принципа $p=\mathfrak{C}$ доказано, что произвольная недискретная метризуемая групповая топология на абелевой групе усиливается до недискретной групповой топологии, в которой каждое нигде не плотное подмножество замкнуто, в частности, замкнуто каждое дискретное подмножество.

Далее все групш предполагаются абелевыми.

Лемма 1. Пусть $(G, \tau)$ - недискретная метризуемая топологическая группа конечного простого периода, $X$ - нигде не плотное подмнохество из $(G, \tau)$, касающееся нуля. Топология $\tau$ усиливается до недискретной метризуемой групповой топологии, в которой множество $Х$ уже не касается нуля. 
ДоКАЗАТЕЛЬСТво. Мы построим в $(G, \tau)$ недискретную подгруппу, дизъюнктную с множеством $X,-$ этого будет достаточно. Пусть $\left\{U_{n}: n<\omega\right\}$ - база окрестностей нуля $(G, \tau), p$ - период $G$. Можно считать, что множество $X \cup\{0\}$ замкнуто. Выберем ненулевой элемент $a_{0} \in U_{0}$ такой, что $\left\langle a_{0}\right\rangle \cap X=\varnothing$, где $\left\langle a_{0}\right\rangle$ - подгруппа, порожденная $a_{0}$. Это можно сделать, так как для каждого ненулевого натурального $n<p$ отображение $x \mapsto n x$ на $(G, \tau)$ является гомеоморфизмом и объединение конечного числа нигде не плотных множеств нигде не плотно. Пусть $V_{1}, W_{1}$ - окрестности нуля $(G, \tau)$ такие, что

$$
\left(\left(\left\langle a_{0}\right\rangle \backslash\{0\}\right)+V_{1}\right) \cap X=\varnothing, \quad \underbrace{W_{1}+\cdots+W_{1}}_{p-1} \subseteq V_{1} .
$$

Выберем ненулевой элемент $a_{1} \in U_{1} \cap W_{1}$ такой, что $\left\langle a_{1}\right\rangle \cap X=\varnothing$. Тогда $\left\langle a_{0}, a_{1}\right\rangle \cap X=\varnothing$. Пусть $V_{2}, W_{2}$ - окрестности нуля такие, что

$$
\left(\left(\left\langle a_{0}, a_{1}\right\rangle \backslash\{0\}\right)+V_{2}\right) \cap X=\varnothing, \quad \underbrace{W_{2}+\cdots+W_{2}}_{p-1} \subseteq V_{2} .
$$

Выберем ненулевой элемент $a_{2} \in U_{2} \cap W_{2}$ такой, что $\left\langle a_{2}\right\rangle \cap X=\varnothing$. Тогда $\left\langle a_{0}, a_{1}, a_{2}\right\rangle \cap$ $X=\varnothing$. Продолжая этот процесс, мы построим недискретную подгруппу $\left\langle a_{0}, a_{1}, \ldots\right.$, $\left.a_{n}, \ldots\right\rangle$.

Лемма 2. Пусть $(G, \tau)$ - недискретная метризуемая топологическая группа, в которой каждая подгруппа конечного периода дискретна. Топология $\tau$ усиливается до недискретной метризуемой групповой топологии, в которой для каэдого ненулевого натурального $n$ отображение $x \mapsto n x$ на G открыто.

ДоказАтЕЛьство. Рассмотрим на $G$ систему множеств вида $n U=\{n x: x \in U\}$, где $n$ - ненулевое натуральное число, а $U$ - окрестность нуля $(G, \tau)$. Эта система удовлетворяет характеристическим свойствам базы окрестностей нуля топологической группы и, следовательно, является базой окрестностей нуля в некоторой групповой топологии $\tau^{\prime}$ на $G$. Очевидно, что $\tau^{\prime}$ метризуема, $\tau^{\prime} \supseteq \tau$ и все отображения на $\left(G, \tau^{\prime}\right)$ вида $x \mapsto n x$ открыты. Недискретность $\tau^{\prime}$ следует из отсутствия в $(G, \tau)$ открытых подгрупा конечного периода.

Лемма 3. Пусть $(G, \tau)$ - недискретная метризуемая топологическая группа, в которой для каждого ненулевого натурального $n$ отображсение $x \mapsto n x$ открыто, $X$-нигде не плотное подмнохсество из $(G, \tau)$, касающееся нуля. Топология $\tau$ усиливается до недискретной метризуемой групповой топологии, в которой множество $X$ уже не касается нуля.

ДокАЗАТЕЛЬСТво. Построим в $(G, \tau)$ нетривиальную сходящуюся к 0 последовательность $\left\{a_{n}: n<\omega\right\}$ такую, что $\left[a_{0}, a_{1}, \ldots, a_{n}, \ldots\right] \cap X=\varnothing$, где

$$
\begin{gathered}
{\left[a_{0}, a_{1}, \ldots, a_{n}, \ldots\right]=\bigcup_{n}\left[a_{0}, a_{1}, \ldots, a_{n}\right]} \\
{\left[a_{0}, a_{1}, \ldots, a_{n}\right]=\left\{x_{0}+x_{1}+\cdots+x_{n}: x_{i} \in\left\{0, \pm a_{i}, \pm 2 a_{i}, \ldots, \pm(i+1) a_{i}\right\}\right\} .}
\end{gathered}
$$

Пусть $\left\{U_{n}: n<\omega\right\}$ - убьвающая база окрестностей нуля $(G, \tau)$. Можно считать, что множество $X \cup\{0\}$ замкнуто. Предположим, что уже выбраны ненулевые элементы 
$a_{0} \in U_{0}, \ldots, a_{n-1} \in U_{n-1}$ такие, что $\left[a_{0}, \ldots, a_{n-1}\right] \cap X=\varnothing$. Пусть $V_{n}, W_{n}-$ симметричные окрестности нуля $(G, \tau)$ такие, что

$$
\left(\left(\left[a_{0}, \ldots, a_{n-1}\right] \backslash\{0\}\right)+V_{n}\right) \cap X=\varnothing, \quad \underbrace{W_{n}+\cdots+W_{n}}_{n} \subseteq V_{n} .
$$

Выберем ненулевой элемент $a_{n} \in U_{n} \cap W_{n}$ такой, что $\pm a_{n}, \pm 2 a_{n}, \ldots, \pm(n+1) a_{n} \notin X$. Тогда $\left[a_{0}, \ldots, a_{n}\right] \cap X=\varnothing$. Такой выбор возможен, так как на $(G, \tau)$ отображения вида $x \mapsto n x, x \mapsto-n x$ открыты, прообраз нигде не плотного множества при открытом отображении нигде не плотен и объединение конечного числа нигде не плотных множеств ниг де не плотно.

Пусть $\tau^{\prime}$ - наибольшая групповая топология на $G$, в которой последовательность $\left\{a_{n}: n<\omega\right\}$ сходится к 0 . Тогда $\tau^{\prime} \supset \tau$ и в топологии $\tau^{\prime}$ множество $\left[a_{0}, a_{1}, \ldots, a_{n}, \ldots\right]$ является окрестностью нуля [7]. Ослабим топологию $\tau^{\prime}$ до недискретной метризуемой топологии $\tau^{\prime \prime} \supset \tau$, в которой множество $\left[a_{0}, a_{1}, \ldots, a_{n}, \ldots\right]$ остается окрестностью нуля. Топология $\tau^{\prime \prime}$ является искомой.

Лемма 4. Пусть $\left\{\tau_{\alpha}: \alpha<\mathfrak{C}\right\}-$ возрастающая $\mathfrak{C}$-последовательность топологий счетного веса на множестве $Y$ такая, что $\tau_{\alpha}=\bigcup\left\{\tau_{\beta}: \beta<\alpha\right\}$, если $\alpha-$ предельный ординал счетной конфинальности. Пусть $X$ - подмножество $Y$, нигде не плотное в топологии $\tau, \tau=\bigcup\left\{\tau_{\alpha}: \alpha<\mathfrak{C}\right\}$. Тогда существует ординал $\gamma<\mathfrak{C}$ такой, что множсество $X$ нигде не плотно в топологии $\tau_{\gamma}$.

ДокАЗАТЕЛЬСТво. Пусть $\tau^{\prime}, \tau^{\prime \prime}$ - произвольные топологии на множестве $Y$ такие, что $\tau^{\prime} \subseteq \tau^{\prime \prime}$. Будем говорить, что подмножество $X \subset Y \tau^{\prime \prime}$-нигде не плотно в топологии $\tau^{\prime}$, если для любого непустого открытого множества $U$ в топологии $\tau^{\prime}$ найдется непустое открытое множество $V \subseteq U$ в топологии $\tau^{\prime \prime}$ такое, что $V \cap X=\varnothing$.

Построим последовательность $\left\{\alpha_{n}: n<\omega\right\}$ счетных ординалов такую, что для каждого $n<\omega$ множество $X \quad \tau_{\alpha_{n+1}}$-нигде не плотно в топологии $\tau_{\alpha_{n}}$, и положим $\gamma=\sup \left\{\alpha_{n}: n<\omega\right\}$.

Основной результат данной заметки мы докажем в предположении комбинаторного принципа $p=\mathfrak{C}$, известного как лемма Буса (см., например, [8]). Согласно $p=\mathfrak{C}$ каждый фильтр на счетном множестве характера $<\mathfrak{C}$ усиливается до последовательности. Иными словами, для каждого фильтра $\varphi$ на счетном множестве $X$ с базой мощности $<\mathfrak{C}$ существует последовательность $\left\{a_{n}: n<\omega\right\}$ элементов из $X$ со следующим свойством: для любого множества $A \in \varphi$ найдется номер $n$ такой, что $\left\{a_{m}: m \geqslant n\right\} \subseteq A$. Комбинаторный принцип $p=\mathfrak{C}$ следует из аксиомы Мартина (МА), которая, в свою очередь, следует из континуум-гипотезы $(\mathrm{CH})$.

Лемма $5(p=\mathfrak{C})$. Каждая недискретная групповая топология на счетной группе характера < $\mathfrak{C}$ усиливается до недискретной метризуемой әрупповой тополо2uu.

ДокАЗАТЕЛЬСТВО. Пусть $\theta<\mathfrak{C},\left\{U_{\alpha}: \alpha<\theta\right\}-$ база окрестностей нуля счетной недискретной топологической групшы $(G, \tau)$. Используя $p=\mathfrak{C}$, построим в $(G, \tau)$ $\theta+1$ цепочку нетривиальных сходящихся к 0 последовательностей $\left\{a_{n}^{\alpha}: n<\omega\right\}, \alpha \leqslant \theta$, такую, что для каждого $\alpha<\theta$

1) $\left|\left\{a_{n}^{\alpha}: n<\omega\right\} \backslash\left\{a_{n}^{\alpha+1}: n<\omega\right\}\right|<\omega$

2) $\left[a_{0}^{\alpha}, a_{1}^{\alpha}, \ldots, a_{n}^{\alpha}, \ldots\right] \subseteq U_{\alpha}$ (см. доказательство леммы 3$)$. 
Затем усилим топологию $\tau$ до недискретной метризуемой групповой топологии, в которой множества $\left[a_{n}^{\theta}, a_{n+1}^{\theta}, \ldots\right], n<\omega$, являются окрестностями нуля.

ТеоремА $(p=\mathfrak{C})$. Произвольная недискретная метризуемая групповая топология на әруппе усиливается до недискретной групповой топологии, в которой каждое нигде не плотное подмножество замкнуто.

ДокАЗАТЕЛьСтво. Пусть $(G, \tau)$ - произвольная недискретная метризуемая топологическая групша. Можно считать, что $G$ счетна. Пусть $\left\{X_{\xi}: \xi<\mathfrak{C}\right\}-$ пересчет всех подмножеств из $G$. Построим возрастающую $\mathfrak{C}$-последовательность $\left\{\tau_{\alpha}: \alpha<\mathfrak{C}\right\}$ недискретных метризуемых групповых топологий на $G$, содержащих $\tau$, которая удовлетворяет следующим условиям:

1) $\tau_{\alpha}=\bigcup\left\{\tau_{\beta}: \beta<\alpha\right\}$, если $\alpha$-предельный ординал счетной конфинальности;

$2)$ для каждого $\xi<\mathfrak{C}$ сушествует $\alpha(\xi)<\mathfrak{C}$ такой, что во всех топологиях $\tau_{\beta}$, где $\alpha(\xi) \leqslant \beta<\mathfrak{C}$, множество $X_{\xi}$ не является нигде не плотным множеством, касаюшимся нуля.

Можно считать, что имеет место один из двух случаев:

a) $(G, \tau)$ содержит открытую подгруппу конечного простого периода;

б) в $(G, \tau)$ каждая подгруппа конечного периода дискретна.

Рассмотрим случай а). В роли $\tau_{0}$ возьмем топологию $\tau$. Фиксируем ненулевой ординал $\alpha<\mathfrak{C}$ и предположим, что для всех $\beta<\alpha$ уже определены $\tau_{\beta}$. Если $\alpha-$ предельньй ординал счетной конфинальности, то $\tau_{\alpha}$ определим условием 1$)$. Если $\alpha-$ предельньй ординал несчетной конфинальности, то, используя лемму 5 , возьмем в роли $\tau_{\alpha}$ любую недискретную метризуемую групповую топологию, содержащую $\bigcup\left\{\tau_{\beta}: \beta<\alpha\right\}$. Пусть $\alpha=\beta+1, X$-первое множество (в $\mathfrak{C}$-последовательности $\left.\left\{X_{\xi}: \xi<\mathfrak{C}\right\}\right)$, котороев топологии $\tau_{\beta}$ нигде не плотно и касается нуля. С помощью леммы 1 усилим $\tau_{\beta}$ до недискретной метризуемой групповой топологии, в которой множество $X$ уже не касается нуля. Эту топологию и возьмем в роли $\tau_{\alpha}$.

Рассмотрим теперь случай б). С помощью леммы 2 усилим топологию $\tau$ до недискретной метризуемой групповой топологии, в которой все отображения вида $x \mapsto n x$ открыты. Эту топологию возьмем в роли $\tau_{0}$. Фиксируем ненулевой ординал $\alpha<\mathfrak{C}$ и предположим, что для всех $\beta<\alpha$ уже определены топологии $\tau_{\beta}$, причем во всех этих топологиях отображения вида $x \mapsto n x$ открыты. Если $\alpha-$ предельный ординал счетной конфинальности, то $\tau_{\alpha}$ определим условием 1$)$. Тогда в $\tau_{\alpha}$ все отображения $x \mapsto n x$ также открыты. Если $\alpha$ - предельньй ординал несчетной конфинальности, то, используя леммы 5 и 2 , возьмем в роли $\tau_{\alpha}$ любую недискретную метризуемую группову топологию, которая содержит $\bigcup\left\{\tau_{\beta}: \beta<\alpha\right\}$ и в которой все отображения вида $x \mapsto n x$ открыты. Пусть $\alpha=\beta+1, X$ - первое множество, которое в топологии $\tau_{\beta}$ нигде не плотно и касается нуля. С помощью лемм 3,2 усилим $\tau_{\beta}$ до недискретной метризуемой групповой топологии, в которой множество $X$ уже не касается нуля и все отображения $x \mapsto n x$ открыты. Эту топологию и возьмем в роли $\tau_{\alpha}$.

Очевидно, что так построенная $\mathfrak{C}$-последовательность $\left\{\tau_{\alpha}: \alpha<\mathfrak{C}\right\}$ удовлетворяет условию 1). Покажем, что она удовлетворяет условию 2). Действительно, фиксируем ординал $\xi<\mathfrak{C}$ и предположим, что для всех $\eta<\xi$ условие 2) вьполняется. Пусть $\gamma=\sup \{\alpha(\eta): \eta<\xi\}$. Если во всех топологиях $\tau_{\beta}$, где $\gamma \leqslant \beta<\mathfrak{C}$, множество $X_{\xi}$ не является нигде не плотным множеством, касающимся нуля, то в роли $\alpha(\xi)$ можно взять $\beta+1$. Если же в некоторой топологии $\tau_{\beta}$ множество $X_{\xi}$ нигде не плотно и касается 
нуля, то в $\tau_{\beta}$ оно является первым из таких множеств и, следовательно, в роли $\alpha(\xi)$ можно взять $\beta+1$.

Положим $\tau^{*}=\bigcup\left\{\tau_{\alpha}: \alpha<\mathfrak{C}\right\}$ и докажем, что в $\left(G, \tau^{*}\right)$ каждое нигде не плотное подмножество замкнуто. Допустим противное. Тогда существует $\xi<\mathfrak{C}$ такой, что множество $X_{\xi}$ в топологии $\tau^{*}$ нигде не плотно и касается нуля. Но по лемме 4 для каждого $\alpha<\mathfrak{C}$ существует $\beta \geqslant \alpha, \beta<\mathfrak{C}$ такой, что множество $X_{\xi}$ нигде не плотно и касается нуля в топологии $\tau_{\beta}$. Получили противоречие с условием 2 ).

ЗАмЕчАниЕ 1 . В работе [9] в предположении $p=\mathfrak{C}$ для каждого натурального числа $n \geqslant 1$ на счетной булевой групе $B$ построены три различные групповые топологии $\lambda_{n}, \rho_{n}, \varkappa_{n}$ с ровно $n$ свободньми сходящимися к 0 ультрафильтрами. Во всех этих топологиях каждое дискретное подмножество замкнуто. Другие примеры недискретных топологических груп, в которых каждое дискретное подмножество замкнуто, были неизвестны. Основной результат данной заметки можно доказать в усиленном варианте (естественно, в предположении $p=\mathfrak{C}$ ): произвольная недискретная метризуемая групповая топология на групе усиливается до недискретной групповой топологии с бесконечным числом сходящихся к 0 ультрафильтров, в которой каждое нигде не плотное подмножество замкнуто.

ЗАмЕчАниЕ 2. Согласно теореме Малыхина в счетной неразложимой топологической групе каждое дискретное подмножество замкнуто. На нигде не плотные подмножества этот результат не распространяется. Для каждого натурального числа $n>1$ топологическая група $\left(B, \varkappa_{n}\right)$ неразложима и в ней существует незамкнутое нигде не плотное подмножество.

\section{СПИСОК ЦИТИРОВАННОЙ ЛИТЕРАТУРЫ}

[1] Сирота С. Произведение топологических групп и экстремальная несвязность // Матем. сб. 1969. T. 79. № 2. C. 179-192.

[2] Малыхин В.И. Экстремально несвязные и близкие к ним группы // Докл. АН СССР. 1975. T. 220. №1. C. 27-30.

[3] Малыхин В.И. Об экстремально несвязных топологических группах // УМН. 1979. Т. 34. №6. C. $59-66$.

[4] Нерешенные задачи топологической алгебры. Кишинев: Штиинца, 1985.

[5] Протасов И. В. Дискретные подмножества топологических групп // Матем. заметки. 1994. T. 55. № 1. С. $150-151$.

[6] Протасов И.В.Абсолютно разложимые группы // Укр. матем. ж. 1996. Т. 48. №3. C. 383-392.

[7] Зеленюк Е. Г., Протасов И. В. Топологии на абелевых группах // Изв. АН СССР. Сер. матем. 1990. Т. 54. № 5. С. 1090-1107.

[8] Малыхин В. И. Топология и форсинг // УМН. 1983. Т. 38. № 1. С. 69-118.

[9] Зеленюк Е. Г. Топологические группы с конечными полугруппами ультрафильтров // Матем. студіi. Праці Львівського матем. т-ва. 1996. Т. 6. 